\title{
MONOTONICITY OF THE SCHWARZ GENUS
}

\author{
PETAR PAVEŠIĆ
}

\begin{abstract}
Schwarz genus $\mathrm{g}(\xi)$ of a fibration $\xi: E \rightarrow B$ is defined as the minimal integer $n$, such that there exists a cover of $B$ by $n$ open sets that admit partial section to $\xi$. Many important concepts, including Lusternik-Schnirelmann category, Farber's topological complexity and Smale-Vassiliev's complexity of algorithms can be naturally expressed as Schwarz genera of suitably chosen fibrations. In this paper we study Schwarz genus in relation with certain type of morphisms between fibrations. Our main result is the following: if there exist a fibrewise map $f: E \rightarrow E^{\prime}$ between fibrations $\xi: E \rightarrow B$ and $\xi^{\prime}: E^{\prime} \rightarrow B$ which induces an $n$-equivalence between respective fibres for a sufficiently big $n$, then $\mathrm{g}(\xi)=\mathrm{g}\left(\xi^{\prime}\right)$. From this we derive several interesting results relating the topological complexity of a space with the topological complexities of its skeleta and subspaces (and similarly for the category). For example, we show that if a CW-complexes has high topological complexity (with respect to its dimension and connectivity), then the topological complexity of its skeleta is an increasing function of the dimension.
\end{abstract}

\section{INTRODUCTION}

In this article we will use the standard definitions and results about the LusternikSchnirelmann category and the topological complexity. Interested reader can refer to 2 for Lusternik-Schnirelmann category, to [3] for topological complexity of a space, and to $[8$ for topological complexity of a map. Note however that we follow the 'non-normalized' convention, so the category and the topological complexity of a contractible space are both equal to 1 .

Our main objective is to explain the relation between the topological complexity of a space and the topological complexity of its skeleta. A well-known result (see [4, Corollary to Theorem 1] or [2, Theorem 1.66]) states that if $X$ is a non-contractible CW-complex, then $\operatorname{cat}(X) \geq \operatorname{cat}\left(X^{(r)}\right)$ where $X^{(r)}$ denotes the $r$-skeleton of $X$. The result is in a sense surprising because $\operatorname{cat}(X)$ is a homotopy invariant of $X$, while the homotopy type of the skeleton can vary for different CW-decompositions of $X$. As a consequence, the above result restricts the homotopy type of skeleta. For example, it implies that every skeleton of a (non-contractible) coH-space is itself a coH-space.

The relation between the category of a space and the category of its skeleta does not extended directly to topological complexity. For example, the topological complexity is 2 for odd-dimensional spheres and 3 for even-dimensional spheres (see [3. Proposition 4.41]). Therefore, if we consider the standard CW-decomposition of $S^{\infty}$, whose skeleta are finite-dimensional spheres, then the topological complexity of skeleta is an alternating sequence of 2's and 3's, while $\mathrm{TC}\left(S^{\infty}\right)=1$. 
In order to understand the causes for different behaviour of two closely related concepts we study certain properties of the Schwarz genus of a fibration (see 10 . of [2, Section 9.3]). In fact, the category and the topological complexity can be both described in terms of the Schwarz genus of suitably chosen maps. Section 2 is dedicated to the study of the relations between genera of fibrations induced by morphisms of fibrations. The main result is Theorem 2.8 it gives sufficient conditions on a morphism between fibrations $\xi$ and $\xi^{\prime}$ over a common base space which imply that $\mathrm{g}(\xi)=\mathrm{g}\left(\xi^{\prime}\right)$. Under similar assumptions but for a morphism between fibrations $\xi$ and $\xi^{\prime}$ over different base spaces we then obtain an inequality $\mathrm{g}(\xi) \leq \mathrm{g}\left(\xi^{\prime}\right)$. Section 3 is split into three subsection in which we apply the general theory to obtain a series of results that compare topological complexity or category of a space to the topological complexity/category of its subspaces.

We will assume throughout this paper that the spaces under consideration are of the homotopy type of a CW-complex and have base-points, and that all maps are base-point preserving. Nevertheless we will systematically omit the base-points from the notation and we will not distinguish notationally between a map and its homotopy class.

\section{Comparison of Schwarz genera}

Let us recall some basic terminology about fibrations (see 9 for more details). A (Hurewicz) fibration is a triple $(E, \xi, B)$, where the space $B$ is the base, $E$ is the total space and $\xi: E \rightarrow B$ is a map that has the homotopy lifting property for maps from arbitrary spaces. A morphism of fibrations is a pair $(f, \bar{f}):(E, \xi, B) \rightarrow\left(E^{\prime}, \xi^{\prime}, B^{\prime}\right)$, where $f: E \rightarrow E^{\prime}$ and $\bar{f}: B \rightarrow B^{\prime}$ are maps, such that the following diagram commutes

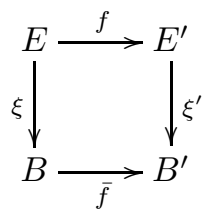

Observe that the map $f$ completely determines the map $\bar{f}$. If $B=B^{\prime}$ and $\bar{f}$ is the identity map, we usually contract the notation and write $f$ instead of $\left(f, 1_{B}\right)$. For every $b \in B$ the preimage $\xi^{-1}(b) \subset E$ is called the fibre of $\xi$ over $b$. In a morphism $(f, \bar{f})$ of fibrations the map $f$ clearly sends the fibre over $b \in B$ to the fibre over $\bar{f}(b) \in B^{\prime}$, so we will occasionally refer to $f$ as a fibrewise map. A map $\sigma: B \rightarrow E$ is a section of $\xi$ if $\xi \circ \sigma=1_{B}$. More generally, if for some $A \subseteq B$, there exists a map $\sigma: A \rightarrow E$, such that $\xi \circ \sigma$ equals the inclusion of $A$ in $B$, we will call it a partial section of $\xi$ over $A$.

In a fibration $(E, \xi, B)$ we will always assume that the base $B$ is path-connected, which in turn implies (cf. 9, Proposition 1.12]) that all fibres of $\xi$ have the same homotopy type. The fibre over the base-point of $B$ will be called the fibre of $\xi$. If $(f, \bar{f}):(E, \xi, B) \rightarrow\left(E^{\prime}, \xi^{\prime}, B^{\prime}\right)$ is a morphism of fibrations, we will denote by $\tilde{f}: F \rightarrow F^{\prime}$ the induced map between the respective fibres.

Following [10] we define the genus $\mathrm{g}(\xi)$ of the fibration $(E, \xi, B)$ as the minimal integer $n$ for which there exist a cover of $B$ by $n$ open sets that admit a partial 
section of $\xi$. In the context of the Lusternik-Schnirelmann category the genus is often called sectional category of $\xi$ (see [2, Section 9.3]).

Observe that genus can be defined for arbitrary maps $\xi: E \rightarrow B$ by requiring that $B$ has a cover by $n$ open sets that admit homotopy sections to $\xi$. If $\xi$ is a fibration, then every homotopy section can be replaced by a strict section, so the two definitions agree. As a matter of fact, most of our results could be easily generalized from fibrations to arbitrary maps. Our goal in this section is to show that certain kind of morphisms between fibrations induce equality between the respective Schwarz genera (see Theorem 2.8). To this end we prove several preparatory lemmas.

Lemma 2.1. If there exists a morphism of fibrations $f:(E, \xi, B) \rightarrow\left(E^{\prime}, \xi^{\prime}, B\right)$, then $\mathrm{g}(\xi) \geq \mathrm{g}\left(\xi^{\prime}\right)$.

Proof. Consider the following diagram

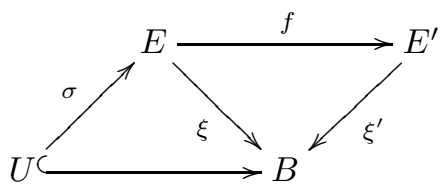

If $\sigma$ is a partial section to $\xi$ over $U$, then $f \sigma$ is a partial section to $\xi^{\prime}$ over $U$, and therefore $\mathrm{g}(\xi) \geq \mathrm{g}\left(\xi^{\prime}\right)$.

Lemma 2.2. Let $f:(E, \xi, B) \rightarrow\left(E^{\prime}, \xi^{\prime}, B\right)$ be a morphism of fibrations, and let $\sigma^{\prime}: B \rightarrow E^{\prime}$ be a section of $\xi^{\prime}$. If a map $\sigma: B \rightarrow E$ satisfies $f \sigma \simeq \sigma^{\prime}$, then $\sigma$ is a homotopy section of $\xi$.

Proof. Straightforward, since $\xi \sigma=\xi^{\prime} f \sigma \simeq \xi^{\prime} \sigma^{\prime}=1_{B}$.

Following [11, Section VII,6.] we will say that a map $f: X \rightarrow Y$ is an $n$-equivalence for $n \geq 1$ if $f$ induces a bijection between the path components of $X$ and $Y$, and if the induced homomorphism on the homotopy groups $f_{\sharp}: \pi_{i}(X) \rightarrow \pi_{i}(Y)$ is an isomorphism for $0<i<n$ and an epimorphism for $i=n$. A canonical example of an $n$-equivalence is the inclusion map $X^{(n)} \hookrightarrow X$ of the $n$-skeleton of a CWcomplex $X$. By [11, Corollary VII,6.23] if $f: X \rightarrow Y$ is an $n$-equivalence, then the induced function $f_{*}:[P, X] \rightarrow[P, Y]$ is bijective for every CW-complex $P$ of dimension $\operatorname{dim}(P) \leq n-1$, and is surjective if $\operatorname{dim}(P) \leq n$.

By analogy, let us say that $f: X \rightarrow Y$ is a homology n-equivalence if the induced homomorphism on the integral homology groups $f_{*}: H_{i}(X) \rightarrow H_{i}(Y)$ is an isomorphism for $0 \leq i<n$ and an epimorphism for $i=n$. By [11, Theorem 7.5.4] an $n$-equivalence is always a homology $n$-equivalence, and the converse holds if $X$ and $Y$ are simply-connected.

Lemma 2.3. Given a morphism of fibrations $f:(E, \xi, B) \rightarrow\left(E^{\prime}, \xi^{\prime}, B\right)$ the fibrewise map $f: E \rightarrow E^{\prime}$ is an n-equivalence if, and only if, the induced map between the respective fibres $\tilde{f}: F \rightarrow F^{\prime}$ is an n-equivalence. 
Proof. Consider the following commutative ladder of exact sequences of homotopy groups of fibrations $(E, \xi, B)$ and $\left(E^{\prime}, \xi^{\prime}, B\right)$ :

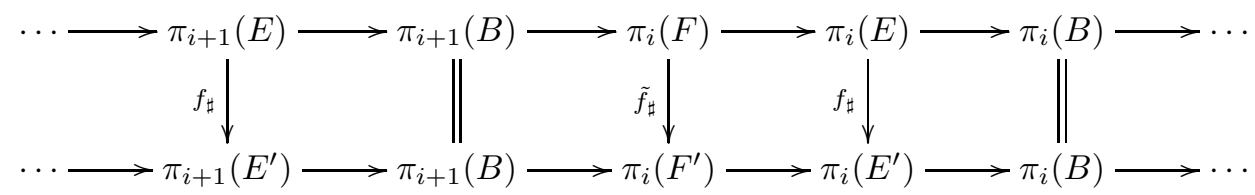

If $\tilde{f}_{\sharp}: \pi_{i}(F) \rightarrow \pi_{i}\left(F^{\prime}\right)$ is an isomorphism for $i<n$, then by the Five-lemma $f_{\sharp}: \pi_{i}(E) \rightarrow \pi_{i}\left(E^{\prime}\right)$ is also an isomorphism for $i<n$. Moreover, if $\tilde{f}_{\sharp}: \pi_{n-1}(F) \rightarrow$ $\pi_{n-1}\left(F^{\prime}\right)$ is an isomorphism and $\tilde{f}_{\sharp}: \pi_{n}(F) \rightarrow \pi_{n}\left(F^{\prime}\right)$ is an epimorphism, then by the Four-lemma $f_{\sharp}: \pi_{n}(E) \rightarrow \pi_{n}\left(E^{\prime}\right)$ is an epimorphism. The converse implication is proved similarly.

Proposition 2.4. Let $f:(E, \xi, B) \rightarrow\left(E^{\prime}, \xi^{\prime}, B\right)$ be a morphism of fibrations such that the induced map $\tilde{f}: F \rightarrow F^{\prime}$ is an n-equivalence for some $n \geq \operatorname{dim}(B)$. Then for every section $\sigma^{\prime}$ of $\xi^{\prime}$ there exists a compatible section $\sigma$ of $\xi$.

Proof. By Lemma 2.2 it is sufficient to find a map $\sigma$ that is a lifting of the map $\sigma^{\prime}$ along $f$ as in the diagram

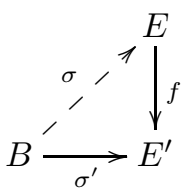

By Lemma 2.3 the map $f: E \rightarrow E^{\prime}$ is an $n$-equivalence. Since $n \geq \operatorname{dim}(B)$, the induced function $f_{*}:[B, E] \rightarrow\left[B, E^{\prime}\right]$ is surjective, which implies that there exists $\bar{\sigma}: B \rightarrow E$, such that $f \bar{\sigma} \simeq \sigma^{\prime}$. By Lemma $2.2 \bar{\sigma}$ is a homotopy section of $\xi$, and $\bar{\sigma}$ can be deformed to a strict section $\sigma: B \rightarrow E$ because $\xi$ is a fibration.

Schwarz [10, II.1] introduced a very useful construction that essentially reduces the computation of the genus to a problem in obstruction theory. Given a fibration $(E . \xi, B)$ with fibre $F$ Schwarz defined the $n$-fold fibrewise join construction (actually called sum in [10]) as a fibration $\left(*_{B}^{n} E, \xi_{n}, B\right)$, where $*_{B}^{n} E$ is a suitable subspace of the $n$-fold join $E * \cdots * E$, and the projection map $\xi_{n}$ is a fibration whose fibre is the $n$-fold join of fibres $F$, which we denote as $*^{n} F$. The main property of Schwarz's construction is stated in the following theorem.

Theorem 2.5 (Schwarz, Theorem 3 in [10]). The fibration $(E, \xi, B)$ has genus $\mathrm{g}(\xi) \leq n$ if, and only if, the $n$-fold fibrewise join $\left(*_{B}^{n} E, \xi_{n}, B\right)$ admits a section.

In other words, $\mathrm{g}(\xi)$ equals the minimal $n$ for which $\xi_{n}$ admits a section. Observe that fibrewise join operation $*_{B}^{n}$ is functorial, i.e. a morphism

$$
f:(E, \xi, B) \rightarrow\left(E^{\prime}, \xi^{\prime}, B\right)
$$

induces a morphism

$$
*_{B}^{n} f:\left(*_{B}^{n} E, \xi_{n}, B\right) \rightarrow\left(*_{B}^{n} E^{\prime}, \xi_{n}^{\prime}, B\right),
$$

whose restriction to the fibres is the usual $n$-fold join of maps $*^{n} \tilde{f}: *^{n} F \rightarrow *^{n} F^{\prime}$. 
Lemma 2.6. Let $X$ be a $(c-1)$-connected space, and let the map $f: X \rightarrow X^{\prime}$ be $a(c+k)$-equivalence $(c, k \geq 0, c+k \geq 1)$. Moreover, let $Y$ be a $(d-1)$-connected space, and let the map $g: Y \rightarrow Y^{\prime}$ be $a(d+k)$-equivalence $(d \geq 0, d+k \geq 1)$. Then $X * Y$ is $(c+d)$-connected, and the map

$$
f * g: X * Y \rightarrow X^{\prime} * Y^{\prime}
$$

is $a(c+d+k+1)$-equivalence.

Proof. Recall the standard homotopy equivalence $X * Y \simeq \Sigma(X \wedge Y)$, which together with the properties of the smash product immediately implies that $X * Y$ is $(c+d)$ connected.

Let us first consider the case where at least one of the spaces $X, Y$ is path-connected, i.e. $c+d \geq 1$. Then $X * Y$ and $X^{\prime} * Y^{\prime}$ are 1-connected, so $f * g$ is a $(c+d+k+1)$ equivalence if, and only if, it is a homology $(c+d+k+1)$-equivalence. To prove the latter it is sufficient to show that $f \wedge g: X \wedge Y \rightarrow X^{\prime} \wedge Y^{\prime}$ is a homology $(c+d+k)$ equivalence. Consider the morphism of the Künneth short exact sequences for the reduced homology of the smash product (see [11, Theorem 5.3.10]):

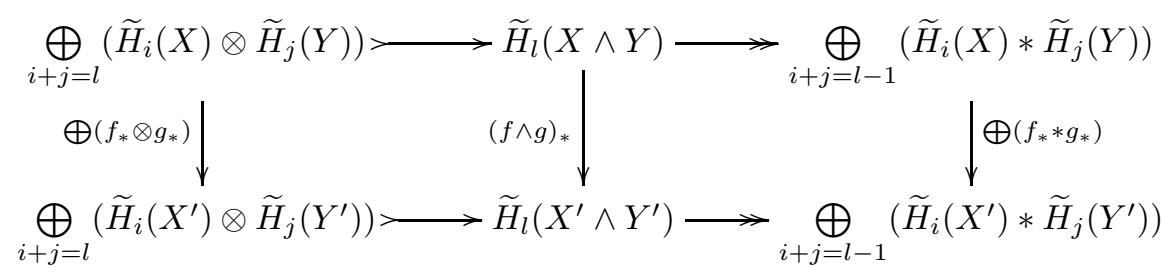

Note that $\widetilde{H}_{i}(X)=\widetilde{H}_{i}\left(X^{\prime}\right)=0$ for $i<c$ and that $\widetilde{H}_{j}(Y)=\widetilde{H}_{j}\left(Y^{\prime}\right)=0$ for $i<d$. Therefore, if $l<c+d+k$, then $\widetilde{H}_{i}(X) \otimes \widetilde{H}_{j}(Y) \neq 0$ only if $c \leq i<c+k$ and $d \leq j<d+k$. Since $f_{*}$ and $g_{*}$ are isomorphisms in that range, we conclude that $\bigoplus\left(f_{*} \otimes g_{*}\right)$ is also an isomorphism. A similar argument shows that $\bigoplus\left(f_{*} * g_{*}\right)$ is an isomorphism, and therefore the middle map $(f \wedge g)_{*}$ is also an isomorphism.

If $l=c+d+k$, then we may follow the same line of reasoning to conclude that the first and the last summand in $\bigoplus\left(f_{*} \otimes g_{*}\right)$ are epimorphisms, while the remaining summands are isomorphisms, therefore $\bigoplus\left(f_{*} \otimes g_{*}\right)$ is an epimorphism. The argument for $\bigoplus\left(f_{*} * g_{*}\right)$ is simpler, because $i+j=l-1$, which implies that all summands are isomorphisms and thus $\bigoplus\left(f_{*} * g_{*}\right)$ is an isomorphism. Four-lemma then implies that $(f \wedge g)_{*}$ is an epimorphism.

If $c=d=0$ (i.e., $X, X^{\prime}, Y$ and $Y^{\prime}$ are disconnected), then $k \geq 1$ and $X * Y, X^{\prime} * Y^{\prime}$ are path-connected but not necessarily simply-connected. To simplify the notation we assume that all spaces have at most countably many components, so let $X_{0}, X_{1}, \ldots$ be the components of $X$, and let $Y_{0}, Y_{1}, \ldots$ be the components of $Y$. Moreover, assume that the base-point $x_{0}$ of $X$ is contained in $X_{0}$, and that the base point $y_{0}$ of $Y$ is contained in $Y_{0}$. By the definition of the smash product we have

$$
X \wedge Y=\left(X_{0} \wedge Y_{0}\right) \amalg\left(\coprod_{i>0} \frac{X_{i} \times Y_{0}}{X_{i} \times y_{0}}\right) \amalg\left(\coprod_{j>0} \frac{X_{0} \times Y_{j}}{x_{0} \times Y_{j}}\right) \amalg\left(\coprod_{i, j>0} X_{i} \times Y_{j}\right),
$$

and there is an analogous description of $X^{\prime} \wedge Y^{\prime}$. Since $f: X \rightarrow X^{\prime}$ induces a bijection between the components of $X$ and $X^{\prime}$, and similarly for $g: Y \rightarrow Y^{\prime}$, it follows that $f \wedge g$ induces a bijection between the components of $X \wedge Y$ and 
$X^{\prime} \wedge Y^{\prime}$. By Seifert-van Kampen's theorem, $f * g: X * Y \rightarrow X^{\prime} * Y^{\prime}$ induces an isomorphism of respective fundamental groups. For higher homotopy groups one have to examine the map induced by $f * g$ between the respective universal covering spaces (or equivalently, consider the induced homomorphisms as homomorphisms of free $\mathbb{Z} G$-modules, where $G=\pi_{1}(X * Y)$ ). The details are straightforward but tedious so we omit them.

Note that the above lemma essentially states that join operation preserves the property that a map is equivalence in the $k$ successive dimensions above the connectivity. Thus we have

Proposition 2.7. Assume that $F$ is $(c-1)$-connected ( $c \geq 0)$ and that $\tilde{f}: F \rightarrow F^{\prime}$ is $a(c+k)$-equivalence for some $k \geq 0$. Then $*^{n} \tilde{f}: *^{n} F \rightarrow *^{n} F^{\prime}$ is an $(n(c+1)+k-1)$ equivalence.

Proof. By inductive application of Lemma 2.6 the $n$-fold join $*^{n} F$ is $(n(c+1)-2)$ connected, hence $\pi_{i}\left(*^{n} \tilde{f}\right)$ is an isomorphism in the following $(k-1)$ dimensions and an epimorphism in dimension $(n(c+1)+k-1)$.

We are now ready to prove the main theorem of this section.

Theorem 2.8. Let $\left(E^{\prime}, \xi^{\prime}, B^{\prime}\right)$ be a fibration with a $(c-1)$-connected fibre $F^{\prime}$. If there exists a morphism of fibrations $f:(E, \xi, B) \rightarrow\left(E^{\prime}, \xi^{\prime}, B^{\prime}\right)$ such that $\tilde{f}$ is a $(c+k)$-equivalence for some $k>\operatorname{dim}(B)-(c+1) \cdot \mathrm{g}\left(\xi^{\prime}\right)$, then $\mathrm{g}(\xi)=\mathrm{g}\left(\xi^{\prime}\right)$.

Proof. By Lemma 2.1 it is always the case that $\mathrm{g}(\xi) \geq \mathrm{g}\left(\xi^{\prime}\right)$, so we only need to prove the converse inequality. By Schwarz's Theorem $n=\mathrm{g}\left(\xi^{\prime}\right)$ implies that the fibration $\xi_{n}^{\prime}$ admits a global section. By naturality of the Schwarz's construction there is a morphism of fibrations $*_{B}^{n} f:\left(*_{B}^{n} E, \xi_{n}, B\right) \rightarrow\left(*_{B}^{n} E^{\prime}, \xi_{n}^{\prime}, B\right)$, whose restriction to the fibres is an $(n(c+1)+k-1)$-equivalence by Proposition 2.7 $\mathrm{By}$ assumptions of the theorem $\operatorname{dim} B \leq n(c+1)+k-1$, so by Proposition 2.4 the fibration $\xi_{n}$ admits a section, hence by Schwarz's Theorem $\mathrm{g}(\xi) \leq n$.

We obtain as an immediate consequence the following comparison of genera of fibrations with different base spaces.

Corollary 2.9. Let $\left(E^{\prime}, \xi^{\prime}, B^{\prime}\right)$ be a fibration with a $(c-1)$-connected fibre $F^{\prime}$. If there exists a morphism of fibrations $(f, \bar{f}):(E, \xi, B) \rightarrow\left(E^{\prime}, \xi^{\prime}, B^{\prime}\right)$ such that $\tilde{f}$ is $a(c+k)$-equivalence for some $k>\operatorname{dim}(B)-(c+1) \cdot \mathrm{g}\left(\xi^{\prime}\right)$, then $\mathrm{g}(\xi) \leq \mathrm{g}\left(\xi^{\prime}\right)$.

Proof. We may decompose the morphism $(f, \bar{f})$ as in the following diagram, where the middle column represents the pullback of $\xi^{\prime}$ along $\bar{f}$, and $f$ is equal to the 
composition $E \rightarrow f^{*} E^{\prime} \rightarrow E^{\prime}$.

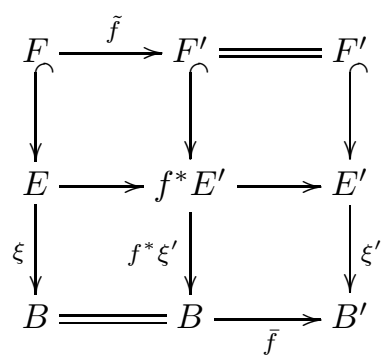

The fibrewise map $E \rightarrow f^{*} E^{\prime}$ satisfies the assumptions of Theorem 2.8 therefore $\mathrm{g}(\xi)=\mathrm{g}\left(f^{*} \xi^{\prime}\right)$. On the other hand, the genus of a pullback of $\xi^{\prime}$ is clearly smaller or equal than the genus of $\xi^{\prime}$, hence $\mathrm{g}(\xi)=\mathrm{g}\left(f^{*} \xi^{\prime}\right) \leq \mathrm{g}\left(\xi^{\prime}\right)$.

\section{Applications}

In this section we are going to compare the values that invariants like the topological complexity or category assume on a space $X$ to the values that the same invariants assume on the skeleta and other subspaces of $X$. Recall that the usual approach to the computation of category and topological complexity is to find suitable upper and lower estimates. General upper estimates are based on dimension and connectivity of the spaces involved (see discussion following Theorem 3.3 for details) while the lower estimates usually rely on the multiplicative structures in cohomology. Although the interval between the upper and lower estimates can be large, in most cases when the values of topological complexity or category are known exactly they are equal (or differ by one) to the general upper bound. Thus, in what follows we will normally begin with a general result and then consider the most interesting special case, when the value of the invariant is close to the general upper bound. Let us first discuss topological complexity of maps.

\subsection{Topological complexity of maps.}

Given a continuous map $u: X \rightarrow Y$ we consider the space $X^{I}$ of all paths $\alpha: I \rightarrow X$, and a map $\xi_{u}: X^{I} \rightarrow X \times Y$ defined as $\xi(\alpha):=(\alpha(0), u(\alpha(1))$. The topological complexity of the map $u$, denoted $\mathrm{TC}(u)$, is defined as the minimal integer $n$ such that there exists an increasing sequence of open subsets

$$
\emptyset=U_{0} \subset U_{1} \subset \ldots \subset U_{n}=X \times Y,
$$

such that each difference $U_{i}-U_{i-1}, i=1, \ldots, n$ admits a continuous partial section to the projection $\xi_{u}$. This concept appeared in [7] where it was used in order to measure the manipulation complexity of a robotic device: $X$ and $Y$ were respectively the configuration space and the working space of a mechanical system (like a robot arm) and $u: X \rightarrow Y$ was interpreted as the forward kinematic map of the system. The theory was further developed in [8]. In particular, if $u$ is a fibration, then by [8, Lemma 4.1] the map $\xi_{u}: X^{I} \rightarrow X \times Y$ is also a fibration. As a consequence, if $u$ is a fibration, then its topological complexity can be expressed in terms of the Schwarz's genus:

$$
\mathrm{TC}(u)=\mathrm{g}\left(\xi_{u}\right)
$$


(see [8, Corollary 4.2]).

Topological complexity of a single space $X$ is clearly equal to the topological complexity of the identity map $1_{X}$. On the other hand, by [8, Corollary 4.8] the category of $X$ can be retrieved as the topological complexity of the path fibration $\mathrm{ev}_{1}: P X \rightarrow X$, where $P X$ denotes the space of all based paths in $X$, and $\mathrm{ev}_{1}$ is the evaluation map that sends a based path $\alpha$ to its end-point $\alpha(1)$.

Theorem 4.9. in [8] allows further simplification, as it shows that $\operatorname{TC}(u)=\mathrm{g}\left(\eta_{u}\right)$, where $\eta_{u}: X \sqcap Y^{I} \rightarrow X \times Y$ is a fibration, whose total space is

$$
X \sqcap Y^{I}=\left\{(x, \alpha) \in X \times Y^{I} \mid f(x)=\alpha(0)\right\}
$$

and $\eta_{u}(x, \alpha)=(x, \alpha(1))$. It is easy to see that the fibre of $\eta_{u}$ is the loop space $\Omega Y$.

Theorem 3.1. Let $(f, \bar{f}):(X, u, Y) \rightarrow\left(X^{\prime}, u^{\prime}, Y^{\prime}\right)$ be a morphism of fibrations, and assume that $Y$ is $(c-1)$-connected and that the map $\bar{f}: Y \rightarrow Y^{\prime}$ is an n-equivalence, for some $n \geq c \geq 1$. If $\operatorname{dim}(X \times Y)<c \cdot\left(\mathrm{TC}\left(u^{\prime}\right)-1\right)+n$, then $\mathrm{TC}(u) \leq \mathrm{TC}\left(u^{\prime}\right)$.

Proof. First of all, observe that $(f, \bar{f})$ determines a morphism of fibration

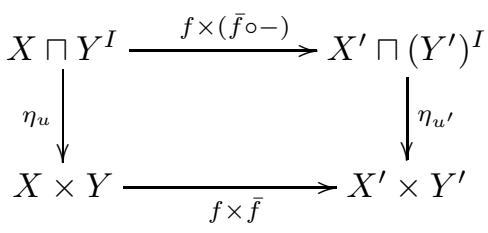

It is easy to check that the induced map between the fibres of $\eta_{u}$ and $\eta_{u^{\prime}}$ is $\Omega \bar{f}: \Omega Y \rightarrow \Omega Y^{\prime}$. By the assumptions of the theorem $\Omega Y$ is $(c-2)$-connected, and $\Omega \bar{f}$ is an $(n-1)$-equivalence. We may apply Corollary 2.9 with $B=X \times Y$ and $k=n-c$ to conclude that $c \cdot\left(\mathrm{g}\left(\eta_{u^{\prime}}\right)-1\right)+n>\operatorname{dim}(X \times Y)$ implies $\mathrm{g}\left(\eta_{u^{\prime}}\right) \geq \mathrm{g}\left(\eta_{u}\right)$. The statement of the theorem follows immediately from the fact that $\mathrm{TC}(u)=\mathrm{g}\left(\eta_{u}\right)$ and $\operatorname{TC}\left(u^{\prime}\right)=\mathrm{g}\left(\eta_{u^{\prime}}\right)$.

\subsection{Topological complexity of spaces.}

From the above we can immediately derive a result on topological complexity of spaces as follows. Let $X$ be a $(c-1)$-connected space and let $f: X \rightarrow X^{\prime}$ be an $n$-equivalence for some $n \geq c \geq 1$. Then we have a morphism of trivial fibrations $(f, f):\left(X, 1_{X}, X\right) \rightarrow\left(X^{\prime}, 1_{X^{\prime}}, X^{\prime}\right)$ so by Theorem 3.1 we obtain

$$
\mathrm{TC}(X) \leq \mathrm{TC}\left(X^{\prime}\right)
$$

provided that $2 \operatorname{dim}(X)<c \cdot\left(\operatorname{TC}\left(X^{\prime}\right)-1\right)+n$. In particular, if $f$ is the inclusion of the $n$-skeleton $X^{(n)}$ into $X$ we have the following result.

Corollary 3.2. Let $X$ be a finite-dimensional $C W$-complex and assume that its $n$ skeleton $X^{(n)}$ is $(c-1)$-connected. If $n<c \cdot(\mathrm{TC}(X)-1)$, then $\mathrm{TC}(X) \geq \mathrm{TC}\left(X^{(n)}\right)$.

If $X \simeq S^{m}$, then its $n$-skeleton is $(n-1)$-connected and at most $n$-dimensional, therefore it is homotopy equivalent to a (possibly empty) wedge of $n$-spheres. If $m$ is odd, then $\operatorname{TC}(X)=2$ and the assumptions of the Corollary are not satisfied, because the dimension of the skeleton cannot be smaller than its connectivity. If $m$ is even, then $\operatorname{TC}(X)=3$ and the assumption of the Corollary is that $n<2 n$, 
which holds for all positive $n$. Therefore if $X \simeq S^{m}$ for $m$ even, then $\operatorname{TC}\left(X^{(n)}\right) \leq 3$ for all $n \geq 1$.

By [6] we know that $\operatorname{TC}(X)=2$ if, and only if, $X$ is homotopy equivalent to an odd-dimensional sphere. Therefore, if $X$ is $(c-1)$-connected and is not homotopy equivalent to a point or an odd-dimensional sphere, then there is always a nonempty range of dimensions, namely $c \leq n<c \cdot(\operatorname{TC}(X)-1)$, for which $\operatorname{TC}(X) \geq$ $\mathrm{TC}\left(X^{(n)}\right)$. If the topological complexity of $X$ is sufficiently large the above range can cover all skeleta above the connectivity of $X$. Thus, by restating the above inequality we obtain the following result.

Theorem 3.3. Let $X$ be a $(c-1)$-connected $C W$-complex, such that $\operatorname{TC}(X) \geq$ $\frac{\operatorname{dim}(X)}{c}+1$. Then $\operatorname{TC}(X) \geq \operatorname{TC}\left(X^{(n)}\right)$ for all $n \geq c$.

Recall that the topological complexity of $X$ is enclosed between the categories of $X$ and of $X \times X$ (see [3, Proposition 4.19]):

$$
\operatorname{cat}(X) \leq \mathrm{TC}(X) \leq \operatorname{cat}(X \times X),
$$

and that for a $(c-1)$-connected $\mathrm{CW}$-complex we have the upper estimate

$$
\operatorname{cat}(X) \leq \frac{\operatorname{dim}(X)}{c}+1 .
$$

(see [2, Theorem 1.50]). In particular, if the category of the space equals the above 'dimension-divided-by-connectivity' estimate (which is often the case), then the assumptions of Theorem 3.3 are satisfied and the topological complexity of $X$ is an upper bound of the topological complexities of its skeleta.

Furthermore, by combining the above estimates we obtain that

$$
\mathrm{TC}(X) \leq \frac{2 \operatorname{dim}(X)}{c}+1
$$

Spaces $X$ for which $\mathrm{TC}(X)$ is equal or close to that upper bound were called spaces with high topological complexity in [5. Examples include all closed surfaces with the exception of the torus, all complex and quaternionic projective spaces, most 3-dimensional lens spaces, configuration spaces, and many other (cf. 3]). Further examples can be obtained by taking finite products of the above. For spaces with high topological complexity we can extend Theorem 3.3 to arbitrary subcomplexes.

Corollary 3.4. Let $X$ be a $(c-1)$-connected $C W$-complex with $\mathrm{TC}(X) \geq \frac{2 \operatorname{dim}(X)}{c}$. Then $\mathrm{TC}(X) \geq \mathrm{TC}(A)$ for every subcomplex $A$ of $X$ containing the $(c+1)$-skeleton of $X$.

Proof. By cellular approximation theorem the inclusion of $A$ in $X$ is at least a $(c+1)$-equivalence. Then $c \cdot(\operatorname{TC}(X)-1)+c+1>2 \operatorname{dim}(A)$ so the statement follows by the discussion preceding Corollary 3.2 .

Still, we have not been able to rule out possible anomalous behaviour, e.g. an increase in topological complexity caused by the removal of a single point The following question would be of some interest to applications:

Question: Does there exist a closed manifold $M$ such that $\mathrm{TC}(M)<\mathrm{TC}(M-x)$ for a single point $x \in M$ ? 
Recall that the $(k+1)$-skeleton of a space $X$ can be obtained as a mapping cone of a single attaching map to its $k$-skeleton, therefore $\operatorname{cat}\left(X^{(k+1)}\right) \leq \operatorname{cat}\left(X^{(k)}\right)+1$. As a consequence the category of skeleta cannot 'jump': if $\operatorname{cat}(X)=n$, then every integer $k=1,2, \ldots, n$ must appear as the category of some skeleton on $X$. The behaviour of the topological complexity can be much more complicated as shown by the sequence of $\operatorname{TC}\left(\mathbb{R} P^{n}\right)$ for $1 \leq n \leq 23$ in [3, p. 122]. In general we have only a coarse estimate [3, Prop. 4.28]

$$
\operatorname{TC}\left(X^{(k+1)}\right) \leq \operatorname{TC}\left(X^{(k)}\right)+\operatorname{cat}\left(X^{(k)}\right)+1 .
$$

Nevertheless, by assuming some control over the category, we are able to show that the topological complexity is an increasing function along the skeleta.

Proposition 3.5. If $\operatorname{cat}(X)=\operatorname{dim}(X)+1$, then

$$
\mathrm{TC}\left(X^{(1)}\right) \leq \mathrm{TC}\left(X^{(2)}\right) \leq \ldots \leq \mathrm{TC}(X) .
$$

Proof. Since $\operatorname{cat}\left(X^{(n)}\right) \leq \operatorname{cat}\left(X^{(n-1)}\right)+1$ for every $n$, it follows that $\operatorname{cat}\left(X^{(n)}\right)=$ $n+1$ for $0 \leq n \leq \operatorname{dim}(X)$. We may thus apply Theorem 3.3 at each stage of the CW-decomposition to show that $\operatorname{TC}\left(X^{(n)}\right) \geq \operatorname{TC}\left(X^{(n-1)}\right)$.

\subsection{Category of maps and spaces.}

The category of a map $u: X \rightarrow Y$, denoted cat $(u)$, is defined as the minimal integer $n$ for which there exists an open covering $U_{1}, \ldots, U_{n}$ of $X$, such that the restrictions $\left.f\right|_{U_{i}}: U_{i} \rightarrow Y$ are null-homotopic (cf. [2, p. 35]). Clearly, the category of a space $X$ is equal to the category of the identity map $1_{X}$. By [2, Proposition 9.18]

$$
\operatorname{cat}(u)=\mathrm{g}\left(\mu_{u}\right)
$$

where $\mu_{u}: X \sqcap P Y \rightarrow X$ is the pull-back of the path-fibration $P Y \rightarrow Y$ along the map $u$, i.e.

$$
X \sqcap P Y=\{(x, \alpha) \in X \times P Y \mid u(x)=\alpha(1)\} \text { and } \mu_{u}(x, \alpha)=x .
$$

Note that we did not require that $u: X \rightarrow Y$ is a fibration, but it is easy to see that the category of a map is equal to the category of its fibrational substitute. As a consequnence, we may assume without loss of generality that $u$ is actually a fibration. The proof of the following theorem is analogous to that of Theorem 3.1 so we omit the details.

Theorem 3.6. Let $(f, \bar{f}):(X, u, Y) \rightarrow\left(X^{\prime}, u^{\prime}, Y^{\prime}\right)$ be a morphism of fibrations, and assume that $Y$ is (c-1)-connected and that the map $\bar{f}: Y \rightarrow Y^{\prime}$ is an n-equivalence, for some $n \geq c \geq 1$. If $\operatorname{dim}(X)<c \cdot\left(\operatorname{cat}\left(u^{\prime}\right)-1\right)+n$, then $\operatorname{cat}(u) \leq \operatorname{cat}\left(u^{\prime}\right)$.

If $u$ and $u^{\prime}$ are taken to be identity maps we obtain a comparison between the category of a space and the categories of its skeleta and subspaces. The following theorem is a generalization of results proved by Felix, Halperin and Thomas [4].

Theorem 3.7. Let $X$ be a $(c-1)$-connected $C W$-complex $X(c \geq 1)$.

(1) If $A$ is a subcomplex of $X$ containing $X^{(n)}(n \geq c)$ and such that

$$
\operatorname{dim}(A)<n+c \cdot(\operatorname{cat}(X)-1),
$$

then $\operatorname{cat}(A) \leq \operatorname{cat}(X)$. 
(2) If $\operatorname{cat}(X) \geq \frac{\operatorname{dim}(X)}{c}$, then $\operatorname{cat}(A) \leq \operatorname{cat}(X)$ for every subcomplex $A \leq X$ containing the $(c+1)$-skeleton of $X$.

(3) if $X$ is not contractible, then $\operatorname{cat}\left(X^{(n)}\right) \leq \operatorname{cat}(X)$ for every $n \geq 1$.

(4) If $X$ is finite-dimensional and $\operatorname{cat}(X)=k$, then

$$
\operatorname{cat}\left(X^{(\operatorname{dim}(X)-k+1)}\right) \leq \ldots \leq \operatorname{cat}\left(X^{(\operatorname{dim}(X)-1)}\right) \leq \operatorname{cat}(X) .
$$

(5) If $\operatorname{cat}\left(X^{(k)}\right)=3$ for some $k$, then

$$
\operatorname{cat}\left(X^{(k)}\right) \leq \operatorname{cat}\left(X^{(k+1)}\right) \ldots \leq \operatorname{cat}(X) .
$$

Proof. If $A$ contains $X^{(n)}$, then the inclusion $A \hookrightarrow X$ is an $n$-equivalence, therefore (1) follows directly from Theorem 3.6.

By the assumptions of (2), cat $(X)$ is close to the 'dimension-divided-by-connectivity' upper bound for the category. The inclusion of $A$ into $X$ is a $(c+1)$-equivalence and $c+1+c \cdot(\operatorname{cat}(X)-1)>\operatorname{dim}(X) \geq \operatorname{dim}(A)$, so the statement is an immediate consequence of (1). Note that the statement remains valid even if $X$ is infinitedimensional.

If $X$ is not contractible, then $\operatorname{cat}(X) \geq 2$ and so (3) follows from (1).

For (4) it is sufficient to observe that, like in the proof of Proposition 3.5. if cat $(X)=$ $k$, then for $n>\operatorname{dim}(X)-k+1$ the $n$-skeleta of $X$ cannot be contractible. Therefore, we can (3) to $n$-skeleta for $n$ in the stated range.

If $\operatorname{cat}\left(X^{(n)}\right) \geq 3$, then $\operatorname{cat}\left(X^{(n+1)}\right) \geq 2$ by [1, Proposition 2.6], and then (3) implies that $\operatorname{cat}\left(X^{(n+1)}\right) \geq \operatorname{cat}\left(X^{(n)}\right) \geq 3$. Thus (5) follows by induction.

\section{REFERENCES}

[1] O. Cornea, Lusternik-Schnirelmann-categorical sections, Annales scientifiques E.N.S., 28 (1995), 689-704.

[2] O. Cornea, G. Lupton, J. Oprea, D. Tanré, Lusternik-Schnirelmann category, Mathematical Surveys and Monographs 103 (American Mathematical Society, Providence, RI, 2003).

[3] M. Farber, Invitation to topological robotics, (EMS Publishing House, Zurich, 2008).

[4] Y. Felix, S. Halperin, J.-C. Thomas, Lusternik-Schnirelmann category of skeleta, Topology Appl. 125 (2002), 357-361

[5] A. Franc, P. Pavešić, Spaces with high topological complexity, Proc. Roy. Soc. Edinburgh, 144 (2014), 761-773.

[6] M. Grant, G. Lupton, J. Oprea, Spaces of topological complexity one, Homology Homotopy Appl. 15 (2013), 73-81.

[7] P. Pavešić, Complexity of the Forward Kinematic Map, Mechanism and Machine Theory 117, (2017), 230-243.

[8] P. Pavešić, Topological complexity of a map, to appear in Homology Homotopy Appl.

[9] P. Pavešić, R.A. Piccinini, Fibrations and their Classification, Research and Exposition in Mathematics, Vol. 33 (Heldermann Verlag, 2013).

[10] A.S. Schwarz, The genus of a fiber space, Amer. Math. Soc. Transl. (2) 55 (1966), 49-140.

[11] E. H. Spanier: Algebraic Topology, (Springer-Verlag, New York 1966).

University of Ljubljana, Faculty of Mathematics and Physics, Luubljana, Slovenia

E-mail address: petar.pavesic@fmf.uni-lj.si 